\title{
Feasibility of diffraction radiation for noninvasive beam diagnostics as characterized in a storage ring
}

\author{
L. Bobb* \\ Diamond Light Source, Oxfordshire OX11 ODE, United Kingdom \\ R. Kieffer, T. Lefevre, and S. Mazzoni \\ CERN, CH-1211 Geneva 23, Switzerland \\ T. Aumeyr and P. Karataev \\ Royal Holloway, University of London, Egham, Surrey TW20 OEX, United Kingdom \\ M. Billing, J. Conway, and J. Shanks \\ Cornell University, Ithaca, New York 14850, USA
}

(Received 28 June 2017; revised manuscript received 5 December 2017; published 5 March 2018)

\begin{abstract}
In recent years, there has been an increasing demand for noninvasive beam size monitoring on particle accelerators. Ideally, these monitors should be cost effective and require little or no maintenance. These monitors should also be suitable for both linear and circular machines. Here, the experimental setup is described in detail, and the results from a diffraction radiation beam size monitor are presented. This monitor has been tested on the Cornell Electron Storage Ring using a $1 \mathrm{~mA}\left(1.6 \times 10^{10}\right.$ particles per bunch) single bunch electron beam at $2.1 \mathrm{GeV}$ energy. Images of the target surface and the angular distribution of the emitted diffraction radiation were acquired at wavelengths of 400 and $600 \mathrm{~nm}$. These measurements are compared to two analytical models.
\end{abstract}

DOI: 10.1103/PhysRevAccelBeams.21.032801

\section{INTRODUCTION}

Diffraction radiation (DR) is the instantaneous emission of photons when a relativistic charged particle moves in the vicinity of a medium. The electric field of the charged particle polarizes the atoms of the medium (or target) which then oscillate, emitting radiation with a very broad spectrum. It should be noted that DR is not produced by a charged particle moving along a continuous boundary; in this case, Cherenkov radiation may be emitted [1].

DR is emitted in two directions from the target: in the direction of the moving charge, known as forward diffraction radiation (FDR), and in the direction of specular reflection, known as backward diffraction radiation (BDR) [2]. BDR is measured for noninvasive beam diagnostics, since it is emitted away from the charged particle given a suitable target tilt angle. The spatial-spectral properties of DR are sensitive to a range of electron beam parameters [3-5].

\footnotetext{
*lorraine.bobb@diamond.ac.uk

Published by the American Physical Society under the terms of the Creative Commons Attribution 4.0 International license. Further distribution of this work must maintain attribution to the author(s) and the published article's title, journal citation, and DOI.
}

The emission of DR is considered to be noninvasive [2]. The energy loss due to DR is much less than the energy of the relativistic charged particle [6]. For this reason, the particle velocity can be treated as constant to a good accuracy, and $\mathrm{DR}$, particularly BDR, can be used for noninvasive beam diagnostics in low background conditions.

The fundamental properties of incoherent DR in the optical wavelength range have been investigated in recent years as a noninvasive counterpart to transition radiation (TR) monitors [7-10]. In the optical wavelength range, the use of diffraction radiation (ODR) as a high-resolution noninvasive diagnostic tool for transverse beam size measurement has been widely investigated, at the Accelerator Test Facility at KEK in Japan [11], at the Free Electron Laser in Hamburg light source at Deutsches Elektronen-Synchrotron [12], and at the Advanced Photon Source at Argonne, USA [13].

Previous DR monitors have been tested as single-pass devices, e.g. with only one DR monitor in a transfer line. In this case, the passage of the charged particle beam through the target aperture is somewhat simplified. For future accelerators, such as the Compact Linear Collider [14], the use of DR monitors would be extended to include both linear and circular sections of the machine where highresolution noninvasive diagnostics are required.

Noninvasive beam size measurement may be provided through the use of synchrotron radiation (SR) monitors 
[15]. However, there are operational limitations that must be considered. Firstly, although these monitors are well suited to electron machines, their use is restricted on proton and ion accelerators where the emission of SR is significantly reduced. Secondly, the footprint of a SR monitor is considerably greater than a DR monitor, and, thirdly, the location is determined by the available source points in the lattice. In some cases, the beta functions at these source points are minimal, which can make beam size measurement using a SR monitor more challenging. The location of a DR monitor is less restrictive and thus could be located to avoid the beam waist.

Laser wire scanners are another alternative for noninvasive monitoring [16]. However, these monitors inherently provide multishot measurements. DR monitors have the capability of performing single-shot beam size measurements. For single-shot monitoring, the only limitation comes from the DR intensity. Through the careful selection of the DR wavelength and the use of intensifiers in the detection system, the light intensity is not expected to be a limiting factor. Furthermore, the costly maintenance of the high power laser in the laser wire system is not applicable to DR monitors.

Installing a DR monitor on a circular machine introduces further advantages and disadvantages not applicable to linear accelerating structures. For example, the target must be redesigned such that it may be retracted for beam injection and aligned with the stored electron beam. Here, new fabrication techniques and beam alignment using direct imaging of the target surface are discussed, as well as optimization of the target to suppress the SR background.

In this paper, the performance of a multipass DR monitor in a storage ring has been benchmarked. The effect due to multiple passes of the beam through a target on the storage ring has been investigated, with a particular focus on the beam lifetime. From this, the potential impact of using several DR monitors along a large scale transfer line or linear accelerator is observed. Furthermore, essential steps in the development of a multipass, simultaneous beam size and position monitor using DR are presented.

\section{GENERAL PROPERTIES OF DIFFRACTION RADIATION}

In this section, the general properties of DR using the ultrarelativistic approximation are summarized.

\section{A. DR distribution}

The DR spectral angular distribution can be calculated using

$$
\frac{d^{2} W}{d \omega d \Omega}=4 \pi^{2} k^{2}\left(\left|E_{x}\right|^{2}+\left|E_{y}\right|^{2}\right),
$$

where the wave number is defined as $k=2 \pi / \lambda, \lambda$ is the wavelength, and $E_{x, y}$ are the horizontal and vertical polarization components, respectively, of the radiation field integrated over the target surface. The total radiation field is derived using the scalar diffraction theory, i.e. when the electron field is introduced as a superposition of individual photons which scatter off the target surface [2,17].

\section{B. Impact parameter}

The emission of DR is dependent on the distance between the charged particle trajectory and the medium. The electric field of a moving charge in a vacuum with velocity $\mathbf{v}$, frequency $\omega$, and energy $E=\gamma m c^{2}$, where $\gamma$ is the Lorentz factor, $m$ is the rest mass of the charged particle, and $c$ is the speed of light, scales as $\exp (-h \omega / \gamma v)$ with distance $h$ in the direction perpendicular to the charged particle velocity. Therefore, DR polarization currents are located in the layer close to the surface of the medium, approximately perpendicular to the charged particle trajectory, and the properties of DR depend strongly on the properties of this layer [2].

The impact parameter $h$, defined as

$$
h \leq \frac{\gamma \lambda}{2 \pi},
$$

describes the condition on the distance from the beam to the slit edge for the emission of DR. This condition is defined by the effective electric field radius of the charged particle $r_{E}=\gamma \lambda / 2 \pi[17]$.

\section{Coherence length}

The radiation formation length or coherence length $L_{f}$ is defined as the region along the particle trajectory where the photon field and the charge particle field overlap one another. The coherence length can be represented as

$$
L_{f}=\frac{\lambda}{\pi} \frac{1}{\left(\gamma^{-2}+\theta_{x}^{2}+\theta_{y}^{2}\right)},
$$

where $\theta_{x, y}$ are the observation angles [2]. For example, if an electron emits two photons at a distance comparable to or smaller than the radiation formation length, those two photons interfere. The photon and electron fields will be completely separated only when the distance along the electron trajectory from the target to the electron is much greater than the radiation formation length [17].

\section{Far field}

The far-field zone is the region at which the angular distribution of DR is observed. The distance from the target to the observation point is assumed to be so large that it is possible to introduce the DR field as a superposition of plane waves of different amplitude emitted by each elementary source of the target. In this case, the Fraunhofer diffraction theory can be used [17]. 
The far field is defined by the condition

$$
\frac{L}{\gamma} \gg \frac{\gamma \lambda}{2 \pi} \rightarrow L \gg \frac{\gamma^{2} \lambda}{2 \pi}
$$

where $L$ is the distance from the target to the detector [17]. From this condition, it is seen that in the far field the distance $L / \gamma$ must be significantly greater than the electric field radius. For the experiments described in this paper, the distance from the target to the detector must be significantly greater than $1 \mathrm{~m}$ to ensure the far-field condition is satisfied.

The angular distribution of DR is emitted in a cone of the order of $\theta=\gamma^{-1}$, where $\theta^{2}=\theta_{x}^{2}+\theta_{y}^{2}$ is the polar observation angle.

\section{E. Prewave zone}

The prewave zone is the region where the far-field condition is not satisfied [18]. In this case, the DR distribution observed is a spatial-spectral distribution; it is not purely angular but includes a spatial contribution determined by the radiation source size. This radiation source size is equal to the electric field radius, which can be treated as the effective electric field radius. For a detector located in the prewave zone, DR photons with different emission angles arrive at the same observation point on the detector plane [17].

If the far-field condition cannot be satisfied due to spatial constraints, the DR angular distribution may be obtained in the prewave zone through the use of a lens with the detector positioned at the back focal plane. A detailed report on the methods of prewave zone suppression can be found in Ref. [17]. In this case, the Fresnel diffraction theory should be used.

\section{THEORETICAL MODELS}

\section{A. Optical diffraction radiation model and the projected vertical polarization component}

It is shown in Ref. [3] that the vertical polarization component is sensitive to the vertical beam size. It is assumed that the electron beam has a Gaussian distribution described by

$$
G\left(\overline{a_{x}}, \sigma_{y}\right)=\frac{1}{\sqrt{2 \pi \sigma_{y}^{2}}} \exp \left[-\frac{\left(\overline{a_{x}}-a_{x}\right)^{2}}{2 \sigma_{y}^{2}}\right],
$$

where $\sigma_{y}$ is the rms vertical beam size, $\overline{a_{x}}$ is the offset of the beam center with respect to the slit center, and $a_{x}$ is the offset of each electron of the beam with respect to the slit center [19].

In Ref. [20], the expression for the ODR vertical polarization component convolved with a Gaussian distribution is shown to be

$$
\begin{aligned}
\frac{d^{2} W_{y}^{\text {slit }}}{d \omega d \Omega}= & \frac{\alpha \gamma^{2}}{2 \pi^{2}} \frac{\exp \left(-\frac{2 \pi a \sin \theta_{0}}{\gamma \lambda} \sqrt{1+t_{x}^{2}}\right)}{1+t_{x}^{2}+t_{y}^{2}} \\
& \times\left\{\exp \left[\frac{8 \pi^{2} \sigma_{y}^{2}}{\lambda^{2} \gamma^{2}}\left(1+t_{x}^{2}\right)\right] \cosh \left[\frac{4 \pi \overline{a_{x}}}{\gamma \lambda} \sqrt{1+t_{x}^{2}}\right]\right. \\
& \left.-\cos \left[\frac{2 \pi a \sin \theta_{0}}{\gamma \lambda} t_{y}+2 \psi\right]\right\},
\end{aligned}
$$

where $t_{x, y}=\gamma \theta_{x, y}, a$ is the target aperture size, $\alpha$ is the fine structure constant, $\theta_{0}$ is the target tilt angle with respect to the particle trajectory, and $\psi=\arctan \left[\frac{t_{y}}{\sqrt{1+t_{x}^{2}}}\right]$ (see Fig. 1). This model is applicable when the TR contribution from the tails of the Gaussian distribution scraping the target is negligible, i.e. approximately $a \geq 4 \sigma_{y}$.

The projected vertical polarization component (PVPC) is a technique which takes the vertical $(y)$ projection of the three-dimensional $\left(\theta_{x}, \theta_{y}\right.$, intensity) DR angular distribution [see Fig. 2(a)]. The vertical projection is obtained by integrating over the horizontal angle $\theta_{x}$ and plotting the resultant intensity as a function of the vertical angle $\theta_{y}$ [see Fig. 2(b)].

The visibility $\left(I_{\min } / I_{\max }\right)$ of the vertical projection is sensitive to the beam size of the electron beam and may be

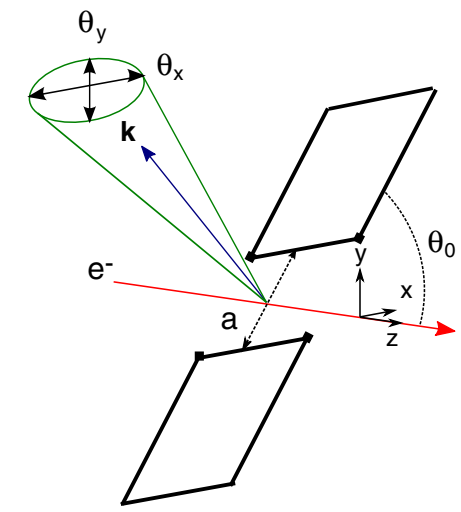

(a)

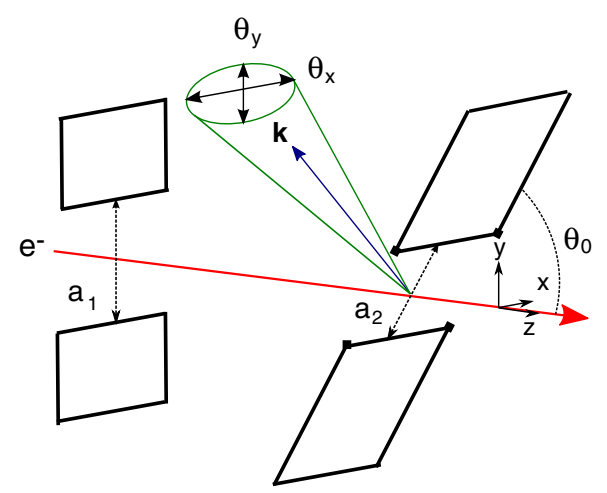

(b)

FIG. 1. Schematic models of the (a) ODR target geometry and (b) ODRI mask and target geometry. 


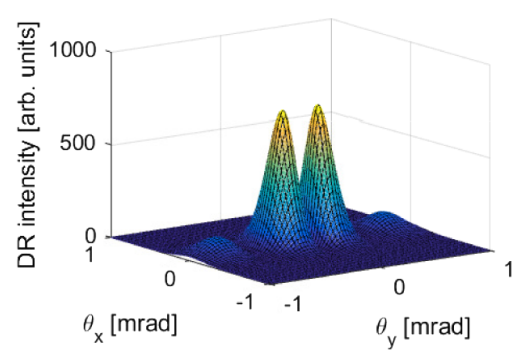

(a)

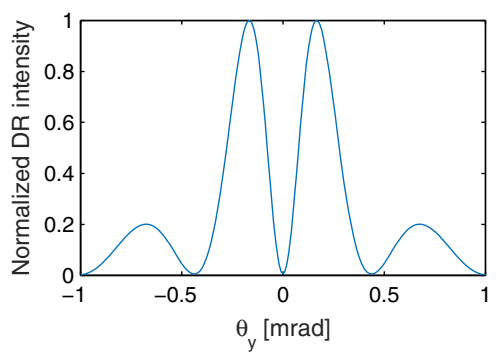

(b)

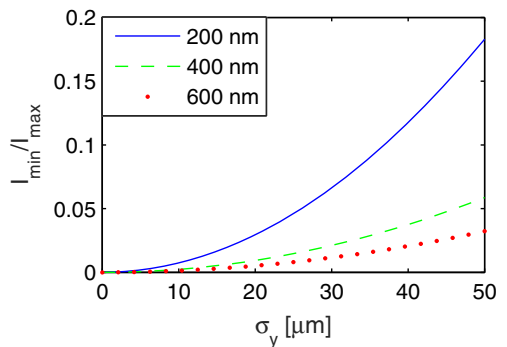

(c)

FIG. 2. A summary of the steps performed in the PVPC technique for beam size measurement: (a) three-dimensional angular distribution of DR, (b) the vertical projection obtained by integrating over the horizontal angle, and (c) the simulated visibility curves for different wavelengths. At a specified wavelength, the visibility measurement obtained from the data is compared to the corresponding simulated visibility curve to obtain the vertical beam size measurement. The parameters are as follows: $a=0.5 \mathrm{~mm}, \overline{a_{x}}=0 \mathrm{~mm}$, $\gamma=4110, \theta_{0}=70^{\circ}$, in (a) and (b) $\lambda=600 \mathrm{~nm}$ and $\sigma_{y}=0 \mu \mathrm{m}$.

measured as shown in Fig. 2(c) [20]. The maximum and minimum intensities of the DR angular distribution must be measured accurately. Measuring the maximum intensity $\left(I_{\max }\right)$ is straightforward, ensuring the detector is not saturated; however, the minimum intensity $\left(I_{\min }\right.$ at $\left.t_{y}=0\right)$ measurement may be limited by background photons. It is also necessary that $I_{\min }$ at $t_{y}=0$ is above the camera noise.

Figure 2(c) shows how the visibility curves at observation wavelengths of 200, 400, and $600 \mathrm{~nm}$ may be obtained from multiple DR angular distribution images over a range of transverse beam sizes. Here it is seen that the sensitivity to the beam size improves at shorter wavelengths, as the change in visibility as a function of the beam size is greater; i.e. the gradient of the visibility curve between different beam sizes is steeper.

Since the vertical projection is used rather that a single line profile, the PVPC method collects more DR photons emitted from the target. In turn, this improves the signal-tonoise ratio and the sensitivity to the beam size, since the minimum intensity of the DR angular distribution is further displaced from zero above the background. This technique has been successfully applied at an extracted beam of the KEK Accelerator Test Facility in Japan [11]. In this paper, this analysis procedure has been applied to data acquired in a circular machine.

\section{B. Optical diffraction radiation interference model}

Generally, in DR experiments a two-slit setup is implemented, where a mask is positioned upstream of the target to reduce unwanted background due to synchrotron radiation. However, it must not be overlooked that the mask is in effect a secondary target and will also emit DR as the beam passes through the mask aperture. It is known that FDR produced by the mask interferes with BDR emitted by the target. Interference occurs between DR emitted by the mask and target when the separation distance between the mask and target is less than the coherence length, which is of the order of 2-3 m using Eq. (3).
The ODR model considers only DR emitted from the target. This model is reasonable provided the interference between the mask and target is small. When this condition is not satisfied, the FDR from the mask must not be ignored, and the optical diffraction radiation interference (ODRI) model should be applied [21].

The DR intensity is obtained from the field component using Eq. (1). Using the ODRI model from Ref. [21], the vertical polarization field component for a single charged particle passing through a slit is represented in the form

$$
\begin{aligned}
E_{y}= & \frac{i e}{4 \pi^{2} c}\left\{\left[\frac{\exp \left[-\left(\frac{a_{1}}{2}+a_{x}-\delta\right)\left(f-i k_{y}\right)\right]}{f-i k_{y}}\right.\right. \\
& \left.-\exp \left(i \Phi_{1}\right) \frac{\exp \left[-\left(\frac{a_{1}}{2}-a_{x}+\delta\right)\left(f+i k_{y}\right)\right]}{f+i k_{y}}\right] \\
& -\exp \left(i \Phi_{0}\right)\left[\frac{\exp \left[-\left(\frac{a_{2}}{2}+a_{x}\right)\left(f-i k_{y}\right)\right]}{f-i k_{y}}\right. \\
& \left.\left.-\exp \left(i \Phi_{1}\right) \frac{\exp \left[-\left(\frac{a_{2}}{2}-a_{x}\right)\left(f+i k_{y}\right)\right]}{f+i k_{y}}\right]\right\},
\end{aligned}
$$

with parameters

$$
\begin{aligned}
k & =\frac{2 \pi}{\lambda}, \\
k_{x} & =k \sin \theta \cos \phi \approx k \theta_{x}, \\
k_{y} & =k \sin \theta \sin \phi \approx k \theta_{y}, \\
\eta & =\frac{k}{\beta \gamma}, \\
f & =\sqrt{k_{x}^{2}+\eta^{2}}, \\
\beta & =\sqrt{1-\frac{1}{\gamma^{2}}}, \\
\Phi_{0} & =\frac{2 \pi d}{\beta \lambda}(1-\beta \cos \theta),
\end{aligned}
$$


where $e$ is the elementary charge, $a_{1,2}$ are the mask and target aperture sizes, respectively, $d$ is the distance between the mask and the target, and $k_{x, y}$ are the components of the wave number $k$ [21].

For a realistic BDR model, one must also take into account the noncoplanarity between the half-planes of the target slit. A noncoplanarity of a few tens of nanometers can produce a significant variation in the $D R$ angular distribution. Therefore, the phase difference $\Phi_{1}=\frac{4 \sqrt{2} \pi \Delta}{\lambda}$, where $\Delta$ is the coplanarity of the target tines in the longitudinal direction [21].

Here, for simplicity, the degree of interference expected given the target $a_{2}$ and mask $a_{1}$ apertures is summarized: $a_{1} \geq 4 a_{2}$ negligible interference of the DR emitted from the mask and target (i.e. the ODR model would still be applicable), $2 a_{2} \leq a_{1}<4 a_{2}$ substantial interference (i.e. the ODRI model should be applied), and $a_{1} \approx a_{2}$ complete destructive interference such that there is no signal. It must be noted that these practical guidelines are applicable only given the observation wavelengths, beam energy, and slit apertures used in this experiment and are not fundamental rules of DR.

One should note that the model above assumes that the entire FDR generated by the mask is reflected from the target. However, this is not entirely correct, because a part of the FDR propagates through the target aperture and is thus not reflected. In this case, a more precise model described in Ref. [22] can be applied.

\section{EXPERIMENTAL SETUP}

\section{A. Cornell Electron Storage Ring Test Accelerator}

The Cornell Electron Storage Ring Test Accelerator (CesrTA) is an electron and positron storage ring used to study ultrarelativistic beam dynamics and beam instrumentation [23]. The layout and parameters of CesrTA are shown in Fig. 3 and Table I, respectively. The DR experiment is located in the L3 straight section of the storage ring. This location was chosen to reduce the synchrotron radiation background from bending magnets upstream. Experiments were performed using a $1 \mathrm{~mA}\left(1.6 \times 10^{10}\right.$ e- per bunch) single bunch electron beam at a beam energy of $2.1 \mathrm{GeV}$. The vertical orbit reproducibility is $10 \mu \mathrm{m}$ turn by turn.

An x-ray beam size monitor (XBSM) [24] was used to measure the vertical beam size and is located at one of the Cornell High Energy Synchrotron Source (CHESS) end stations. The visible beam size monitor (VBSM) [25] was used to measure the horizontal beam size $\sigma_{x}$ and is located in the L3 straight section approximately $10 \mathrm{~m}$ upstream of the DR target. For beam size measurements at the DR target location, measurements from the XBSM and VBSM were scaled using the beta functions. The error on the beta functions is on the order of $2 \%$. The turn-by-turn variation

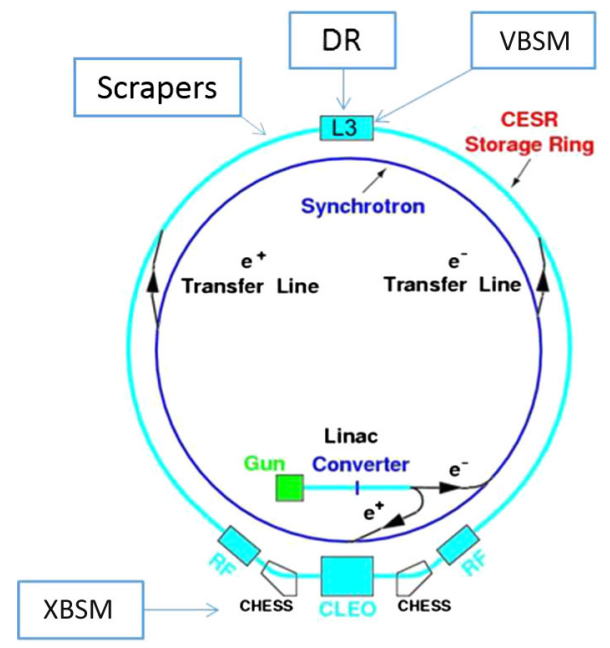

FIG. 3. A schematic of the layout of CesrTA [23].

in the beam size as measured by the XBSM is approximately $\pm 2 \mu \mathrm{m}$ over a 1024 turn acquisition.

A group of skew quadrupoles was used to create a closed vertical dispersion bump through a set of damping wigglers to introduce vertical emittance while preserving the global coupling. Using this group, the vertical beam size at the DR target was varied from 13 to $52 \mu \mathrm{m}$. The horizontal beam size was approximately $490 \mu \mathrm{m}$.

\section{B. DR vacuum chamber}

An overview of the DR vacuum chamber and mechanisms is shown in Fig. 4. The DR chamber is approximately $300 \mathrm{~mm}$ long with respect to the electron beam orbit. The design of the vacuum chamber had to incorporate the DR instrumentation used during CesrTA runs and also a replacement chamber for high current CHESS operation. The replacement chamber is designed to minimize the

TABLE I. Parameters of CesrTA [24].

\begin{tabular}{ll}
\hline \hline Parameter & \multicolumn{1}{c}{ Value } \\
\hline Circumference & $768.4 \mathrm{~m}$ \\
Circulation time & $2.563 \mu \mathrm{s}$ \\
Circulation frequency & $390.1 \mathrm{kHz}$ \\
Beam energy & $2.085(1.5-5.3) \mathrm{GeV}$ \\
Species & $e^{+}$or $e^{-}$ \\
rf frequency & $500 \mathrm{MHz}$ \\
Harmonic number & 1281 \\
Bunch spacing & $\geq 4 \mathrm{~ns}$ \\
Bunch population & $0.1-10 \times 10^{10}$ \\
Number of bunches per turn & $\leq 600$ \\
Horizontal emittance & $2.6 \mathrm{~nm} \mathrm{at} 2.1 \mathrm{GeV}$ \\
Vertical emittance & $\geq 10 \mathrm{pm}$ \\
Longitudinal bunch length (rms) & $10-15 \mathrm{~mm}$ \\
Horizontal beam size (rms) & $170-300 \mu \mathrm{m}$ \\
Vertical beam size (rms) & $10-100 \mu \mathrm{m}$ \\
\hline \hline
\end{tabular}




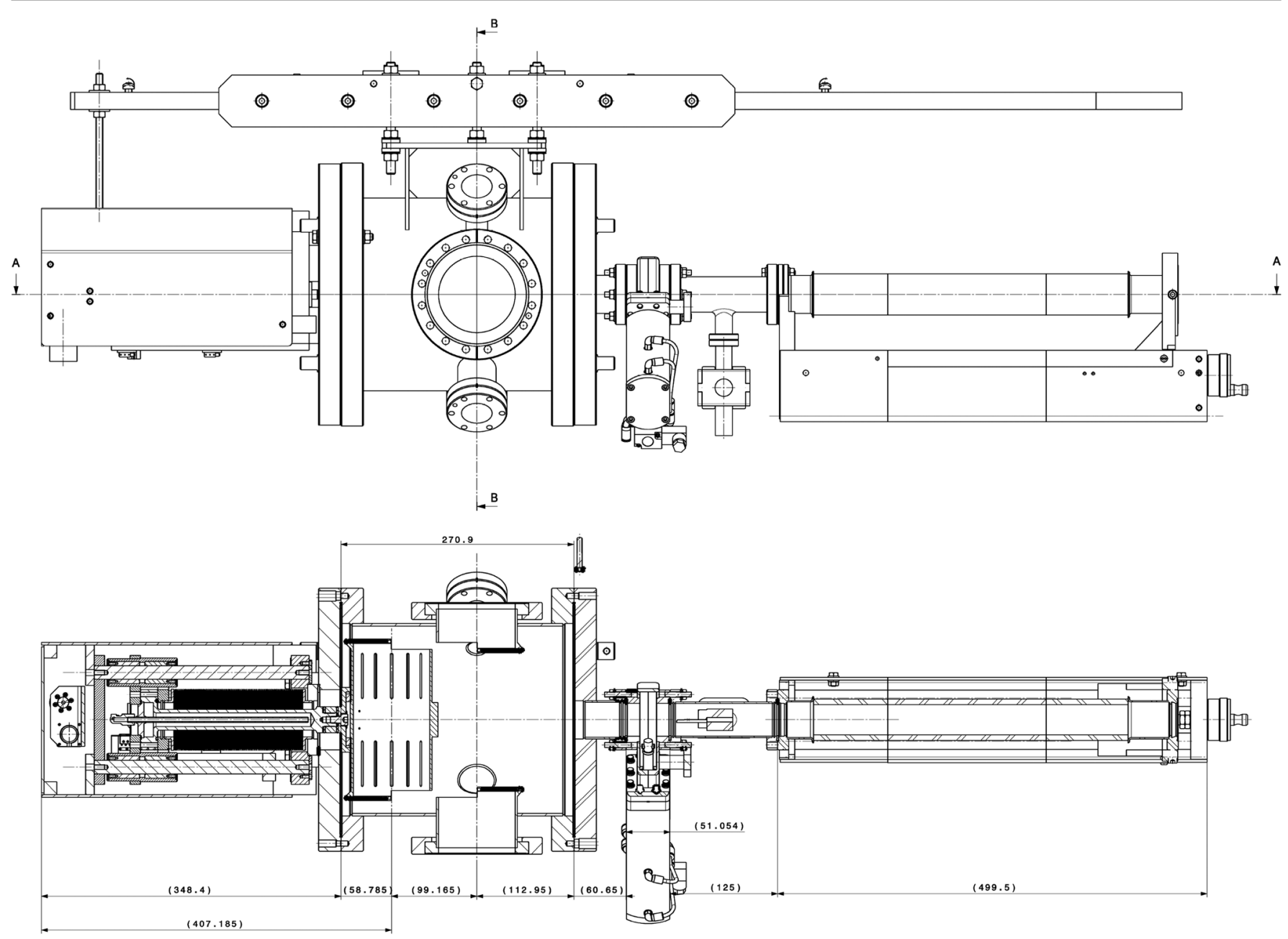

FIG. 4. A technical drawing of the vacuum chamber as viewed from the upstream beam port (top) and from above (bottom) showing the replacement chamber (left) and target mechanism (right) by N. Chritin.

higher order mode loss for the stored beams as they pass through the relatively large vacuum chamber cavity.

On the opposite side of the chamber is the target mechanism. This mechanism has two degrees of freedom: translation in or out and rotation about the insertion axis. Translation is required to insert and retract the target from the beam. Rotation is required to align the BDR with the axis of optical system. The ultrahigh vacuum ZTR3070W translator from VG Scienta was chosen. This translator is stepper motor driven with a $300 \mathrm{~mm}$ motion range and can be mounted in any orientation. It is bakeable to $230^{\circ} \mathrm{C}$. For the DR experiment, the translator was mounted horizontally; therefore, the ZTRST support tube was included to increase the stability of the sample and prevent sagging of the bellows. The ZTRRB rotary drive accessory was included to support the rotary drive shafts over the travel range.

Between the chamber and the target mechanism is a manual gate valve and holding chamber. Without compromising the storage ring vacuum, the target and mask assembly can be retracted and replaced.
Three viewports have been incorporated in the design of the DR chamber. The viewport at the top of the DR chamber in Fig. 4 allows the BDR from the target to enter the optical system for detection. The viewport observation angle is $40^{\circ}$ relative to the charged particle beam trajectory about the target position at the center of the vacuum tank. Directly opposite this viewport, beneath the chamber, is another viewport for visual checks of the target condition and alignment. It should also be noted that this viewport could be used for BDR observation using the counterrotating positron beam in the storage ring. A third flange is available for an additional viewport; however, this was not necessary. Instead, rf probes were connected to measure the efficiency of the replacement chamber.

For the DR window at the top of the DR chamber, an excimer UV-grade fused silica viewport (Vaqtec part number CF40 3-FS-0116) with a view diameter of $36 \mathrm{~mm}$ was chosen. Transmission greater than $85 \%$ is obtained for wavelengths from $200 \mathrm{~nm}$ to $1 \mu \mathrm{m}$. A deep UV-grade fused silica viewport (3-FS-0108) with reduced transmission at shorter wavelengths was chosen for the 
second viewport. Transmission at UV wavelengths for this viewport was not required, since this location is primarily used for visual hardware checks.

Two CESR beam position monitors (BPMs) are in close proximity to the DR target location. Directly attached to the vacuum chamber, approximately $300 \mathrm{~mm}$ upstream of the DR target, is a four-button beam position monitor. This BPM is read out continuously during the DR experiment and is labeled "B48AW." Another BPM is located $300 \mathrm{~mm}$ downstream of the DR target in the electron beam direction, labeled "B48W." This BPM is a member of the normal CESR orbit system and is triggered to acquire turn-by-turn beam orbits. The BPM resolution is $10 \mu \mathrm{m}$ [26].

\section{Optical system}

A compact design was chosen for simple alignment and installation in the storage ring tunnel as shown in Fig. 5. The length of the optical system (mirror to detector) is $<1 \mathrm{~m}$. Considerations were made in the positioning and radiation hardness of the camera due to the close proximity to the beam pipe. The optical system is raised above the radial plane of the storage ring such that the secondary emissions due to SR incident on the camera were reduced.

A dual purpose optical system has been developed for the DR monitor. Direct imaging of the target surface is used for alignment of the electron beam in the target aperture (see Sec. VI A) and beam position monitoring [27]. In the imaging setup, an achromat doublet lens (AC508-150-A) provided by Thorlabs is inserted into the optical path.

The angular distribution of the emitted BDR from the target is required for vertical beam size measurement [11]. Because of its compact length, the optical system is within the prewave zone. As described in Sec. II E, a lens must be used in conjunction with the camera being positioned in the back focal plane to obtain the DR angular distribution [17].
For this purpose, a plano-convex lens (LA4782) from Thorlabs was selected.

The lenses are mounted on Thorlabs flippers so that they can be inserted and removed from the optical path remotely. The details of the optical system are summarized in Table II.

Directly after the DR viewport, a deep UV aluminum mirror (DUVA-PM-2037-UV) from CVI Melles Griot is located. The mirror is mounted on a remotely controlled, motorized stage from Zaber (ZABT-MM2-KT04).

To select different observation wavelengths, narrowband filters with $(10 \pm 2) \mathrm{nm}$ bandwidth from Andover Corporation were chosen. These filters are one of the few components that are not remotely controlled. The filter is installed in a fixed mount in the optical system.

As discussed in Sec. III, the vertical beam size can be determined from the vertical polarization component of the BDR. Although the horizontal component is suppressed by the target geometry, it is still present in the emitted DR. Therefore, a polarizer is included in the optical system. Two polarizers have been tested: a Glan-laser prism (440-2020-M2P) by Eksma Optics and a linear polarizer (LPVISE100-A) from Thorlabs. The Glan-laser polarizer is made of natural calcite with an operating wavelength range of $220 \mathrm{~nm}$ to $2.3 \mu \mathrm{m}$. The extinction ratio is $1: 10^{-5}$. The linear polarizer operates over the 400 to $700 \mathrm{~nm}$ wavelength range with an extinction ratio of $1: 10^{-3}$.

The detector of the optical system is a gated intensified CCD ProxiKit Package camera by Proxivision mounted on a translation stage. Images are acquired with a 12 bit dynamic range and 1390 pixels $\times 1038$ pixels resolution, where the pixel size is $6.45 \mu \mathrm{m} \times 6.45 \mu \mathrm{m}$. An 18:11 fiber taper connects the intensifier to the CCD sensor. The Proxikit Package is a modular setup where each module is chosen to meet the experiment specification.

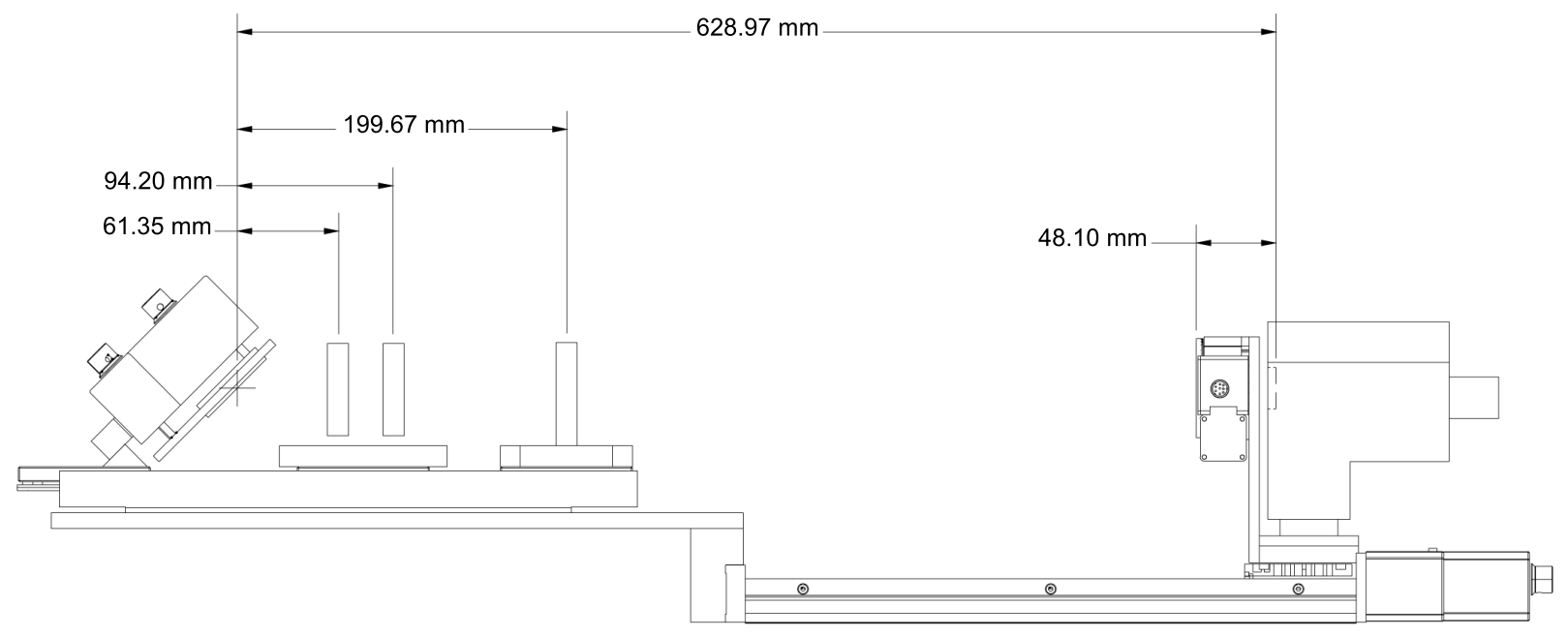

FIG. 5. A technical drawing of the optical system. From left to right is the folding mirror in the motorized holder, the lenses on flip stages for target imaging and angular distribution observation, the fixed bandpass filter, the Glan-laser polarizer in a rotation stage, and the camera mounted on a translation stage for the two observation positions. 
TABLE II. Summary of the optical system parameters.

\begin{tabular}{lcc}
\hline \hline Parameter & Imaging setup & Angular setup \\
\hline Clear aperture (diameter) & $50.8 \mathrm{~mm}$ & $50.8 \mathrm{~mm}$ \\
Focal length & $150 \mathrm{~mm}$ & $500 \mathrm{~mm}$ \\
Wavelength & $400-700 \mathrm{~nm}$ & $185-2100 \mathrm{~nm}$ \\
Magnification & -0.611 & $\cdots$ \\
Angle per pixel & $\cdots$ & $0.0211 \mathrm{mrad}$ \\
\hline \hline
\end{tabular}

\section{TARGET AND MASK FABRICATION TECHNOLOGIES}

Previous DR experiments were installed on linear machines [4-6,21]. A typical target in these tests consisted of a screen similar to those used for optical transition radiation with the modification of a circular or rectangular hole. On circular machines, the target must be retracted during the injection of the beam to the storage ring and then inserted to the stable beam. Therefore, the targets used for DR studies on circular machines must be modified further to have a forklike shape.

\section{A. Coplanarity, roughness, flatness, and aperture size}

As aforementioned in Sec. III B, a good coplanarity between target tines is essential to observe the symmetrical angular distribution needed for beam size measurement [17]. A coplanarity smaller than a tenth of the DR wavelength is required to ensure the angular distribution is sufficiently symmetrical. Producing a $30 \mathrm{~mm}$ long, forkshaped target with a coplanarity less than $50 \mathrm{~nm}$ at the extremities of the target tines is a delicate task (see Fig. 6). Two different techniques were investigated to produce targets which could satisfy these constraints. In addition to the coplanarity specification, the roughness and aperture size must be also controlled during fabrication to avoid distortions in the observed DR angular distribution.

\section{Chemical etching}

Chemical etching is a process where $\approx 1.4 \mathrm{~mm}$ thick, crystalline, optically polished silicon wafers are treated with an etchant to a desired shape. This etchant is traditionally an acidic mixture [28].

Initially, four chemically etched targets were made, two of which had $1.0 \mathrm{~mm}$ apertures and two of which were

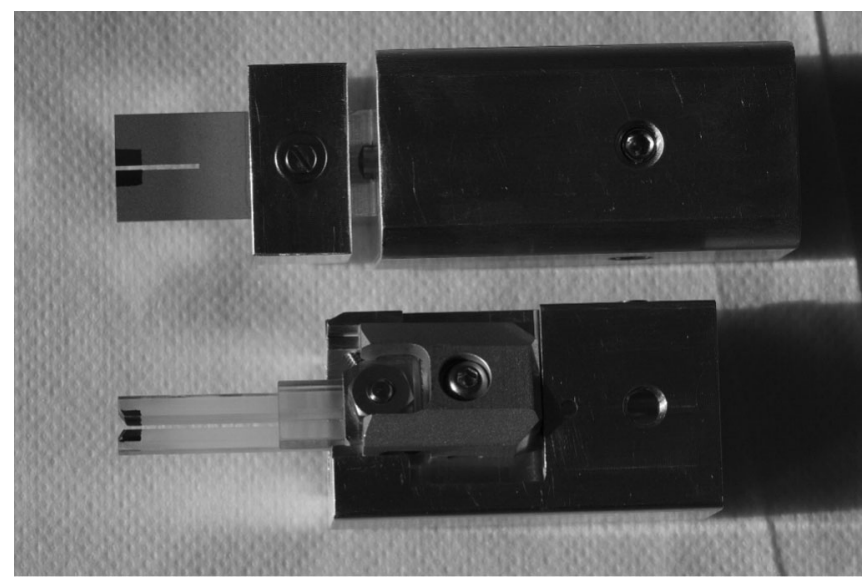

FIG. 6. Silicon etched target (top) and molecular adhesion target (bottom).

stepped targets with 0.5 and $1.0 \mathrm{~mm}$ apertures. The roughness, aperture size, and coplanarity of these targets were measured using the instruments listed in Table III. The VEECO-NT 3300 is a noncontact, optical profiler used to measure the roughness and flatness of samples by interferometry. The MAHR Wegu OMS 600 is a 3D optical coordinate measurement machine which employs multisensor technology.

The metrology results are summarized in Table IV. From these targets, it was found that the aperture size could be produced to within $3 \mu \mathrm{m}$ of the specification. The average roughness was $<2.5 \mathrm{~nm}$. Specular reflection will occur provided the surface roughness is small compared to the wavelength of the incident rays; i.e. the wavelets will interfere constructively in only one direction. Based on these results, a high reflectivity was expected at visible-UV wavelengths.

From this first batch, it was found that the flatness and coplanarity of the tines worsened towards the open, unconstrained end of the target. With this batch of targets, the coplanarity requirement to observe symmetrical lobes in the angular distribution of DR could not be fulfilled. Thus, the alternative technique of molecular adhesion was investigated.

At a later stage, the process of chemical etching was revisited. Thanks to the collaboration with the center of micronanotechnology in the EPFL Lausanne, a dedicated

TABLE III. Metrology instrumentation used to measure the roughness, flatness, and aperture size of the targets at room temperature.

\begin{tabular}{lccc}
\hline \hline & Roughness & Flatness & Distance \\
\hline Instrument & Roughness tester & Roughness tester & MAHR Wegu \\
& VEECO-NT 3300 & VEECO-NT 3300 & OMS 600 \\
Optical zoom & $\times 20$ & $\times 2.5$ & $\times 40$ \\
Optical lens & $\times 1$ & $\times 0.5$ & $\ldots$ \\
Estimated uncertainty & $10 \%$ & $10 \%$ & $\pm 2 \mu \mathrm{m}$ \\
\hline \hline
\end{tabular}


TABLE IV. Measured target parameters from the different methods of fabrication.

\begin{tabular}{lcccc}
\hline \hline & $\begin{array}{c}\text { Average roughness } \\
{[\mathrm{nm}]}\end{array}$ & $\begin{array}{c}\text { Average aperture } \\
\text { size deviation from } \\
\text { specification }[\mu \mathrm{m}]\end{array}$ & Flatness $[\mu \mathrm{m}]$ & $\begin{array}{c}\text { Coplanarity } \\
\text { of tines }[\mu \mathrm{m}]\end{array}$ \\
\hline Chemical etching batch 1 & $<2.5$ & 3.0 & $<10$ & $0.1-10$ \\
Molecular adhesion & $\ldots$ & 3.0 & $<0.04$ & $<0.05$ \\
Chemical etching batch 2 & $<3.8$ & 18.0 & $<0.05$ & $<0.05$ \\
\hline \hline
\end{tabular}

procedure was found to produce the targets. The $\mathrm{KOH}$ anisotropic chemical etching used in the microfabrication process allows the machining of crystalline silicon wafers with almost no mechanical constraint applied to the substrate. In this procedure, the photolithography masks were precisely aligned with respect to the crystalline structure of the wafer to produce very long and narrow slits with a nanometric knife sharp edge over a few millimeters length without bending the substrate [29]. The targets were fabricated with $10 \mathrm{~nm}$ coplanarity after sandblasting as shown in Fig. 7.

\section{Molecular adhesion}

Bonding by molecular adhesion is a technique that enables two substrates having polished surfaces to adhere

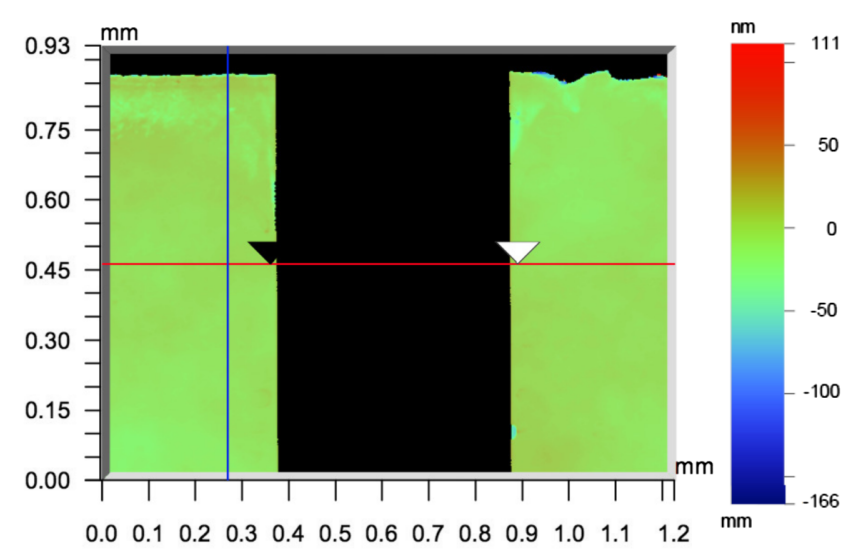

(a)

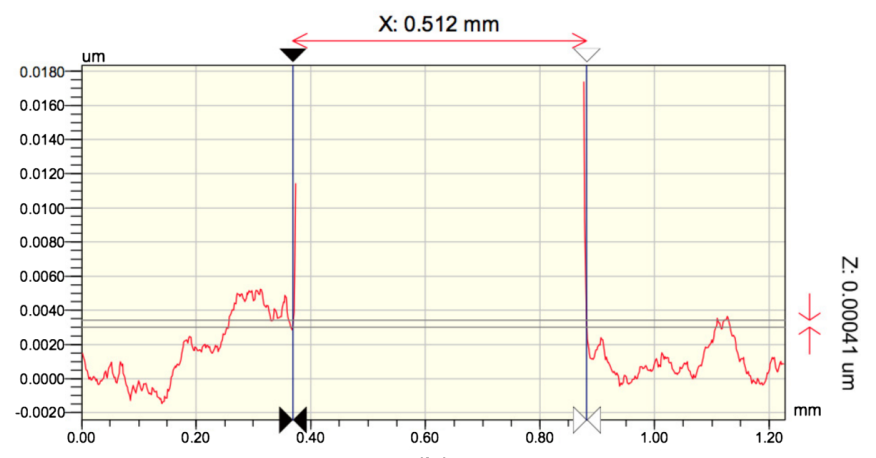

(b)

FIG. 7. Measured (a) flatness and (b) aperture size of a chemically etched and sandblasted target from batch 2 . to one another, without the application of an adhesive [30]. The upper and lower tines of the target are machined and polished separately in sets. The tines are then paired together in all variations to identify which upper or lower pairs result in the best coplanarity and attached to a flat mounting block. In effect, the molecular adhesion target consists of three individually machined pieces: two tines and the mounting block.

Two sets of targets were produced using fused silica $\left(\mathrm{SiO}_{2}\right)$ and silicon $(\mathrm{Si})$ with apertures of 0.5 and $1 \mathrm{~mm}$. An aluminum and chromium coating was applied to the $\mathrm{SiO}_{2}$ targets to enhance the reflectivity at visible and UV wavelengths. Chromium was included with aluminum in the coating to improve the tensile strength.

While chemically etched components have been widely used in physics applications, molecular adhesion is a relatively new technique and, as such, is not currently well understood in accelerator environments. For example, the fragility of the molecular bond with thermal heating due to scattered electrons in the storage ring was one concern. To address this risk, a mounting clamp was designed to surround the target assembly as a precaution should the molecular bonds break. In this event, the individual pieces remain mounted to the target holder and cannot fall into the vacuum chamber.

Metrology measurements for the molecular adhesion targets were performed by the manufacturer and are included in Table IV. The achieved aperture size is comparable to that of the chemically etched targets. Generally, the coplanarity of the molecular adhesion targets was an order of magnitude better than the chemically etched counterpart.

\section{B. Synchrotron radiation suppression}

Regardless of the method of fabrication, the majority of the targets were fully coated with a reflective metallic layer (aluminum $30 \mathrm{~nm}$ ) and a protective layer (magnesium fluoride $\mathrm{MgF}_{2} 10 \mathrm{~nm}$ ) to enhance the photon yield. The DR photons are produced on the tine edges, in close proximity to the beam, whereas the SR background generated by upstream magnetic elements is usually distributed across the target surface (see Fig. 8). Switching the optical system from direct imaging of the target surface to the setup used for observation of the DR angular distribution means it is no longer possible to separate the two sources of radiation, DR and SR, from the recorded angular distribution. 


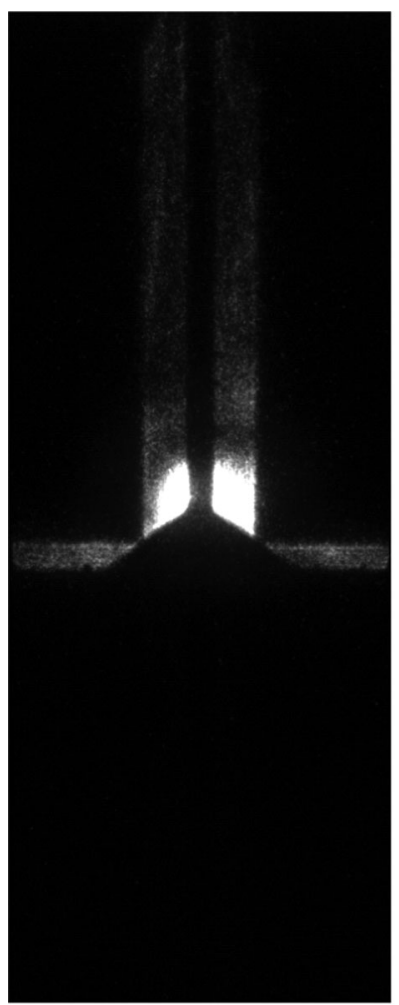

(a)

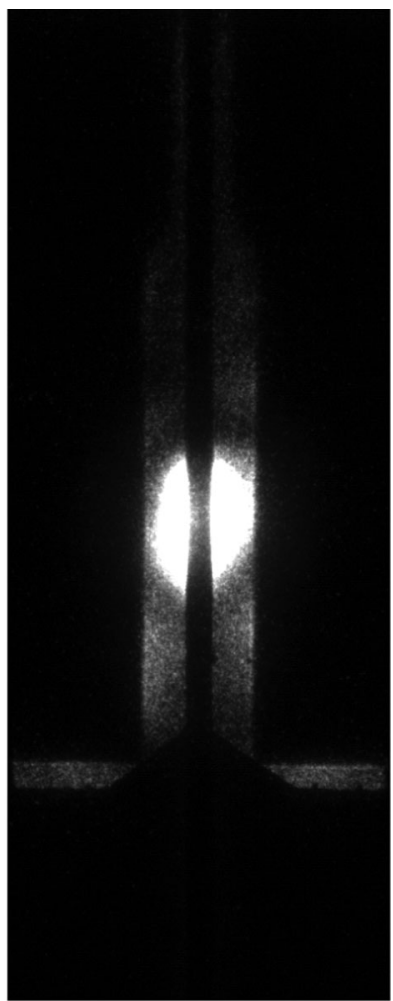

(b)

FIG. 8. Images of the target surface taken during the insertion of the target around the stored electron beam. Because of the orientation of the optical system, the images are rotated by $90^{\circ}$; i.e. the horizontal plane along which the target is inserted is parallel with the target tines, and the width of the target aperture is in the vertical direction. The local disks of DR are clearly visible at the slit edges. The fairly uniform illumination due to the SR background passing through the mask aperture is also seen.

\section{Mask}

A silicon carbide mask was used to reduce the contribution of SR to background and was mounted upstream of the target as shown in Fig. 9. The mask efficiency has been well demonstrated in Ref. [31]. The mask edges are not etched, since it is oriented perpendicular to the incident electron beam. Typically, the mask aperture was 4 times larger than the target aperture to minimize interference effects. For the molecular adhesion targets, a stepped mask

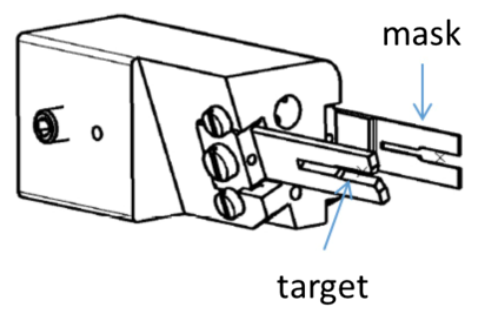

FIG. 9. Target holder with mask and target mounted (by N. Chritin).

was used with aperture sizes a factor of 4 and 2 larger than the target aperture to allow the observation without and with interference effects between FDR from the mask and BDR from the target, respectively.

\section{Target surface optimization}

In a two-slit setup, the mask profile follows that of the target. Therefore, there is a strip along the full length of the target tines which is not shielded from SR. To further reduce the SR background, alternative masking methods, such as sputtering the reflective coating in a specified region only and sandblasting the polished surfaces of the target directly, were investigated.

The mirrored reflective area surrounding the target aperture was reduced to a square with a length 10 times the effective field radius. Microsandblasting techniques were used to make the surface surrounding the mirrored area fully diffusive. The reflectivity was measured with a reflectometer; taking into account the aperture of the optical line, the amount of SR light reflected from the diffusive surface is reduced by 3 orders of magnitude. In Fig. 10, postsandblasting, the reflectivity is reduced from $90 \%$ to $0.1 \%$ at visible wavelengths.

\section{Closed orbit bumps}

In addition to the SR suppression through the design of the target and mask, adjustments were made to the vertical orbit to reduce the SR incident on the target. The nearest bending magnet, SB48E, whose end is located $14.2 \mathrm{~m}$ upstream of the target, has a bending radius of $140.6 \mathrm{~m}$. Nominally, the SR emitted from this magnet directly illuminates the target. However, by adjusting vertical bumps through this magnet, the emitted SR was directed away from the target, thus minimizing the total SR intensity incident on the target surface and improving the signal-tonoise ratio.

Further upstream, the SR from bending magnet HB47E indirectly illuminates the target via reflections on the vacuum

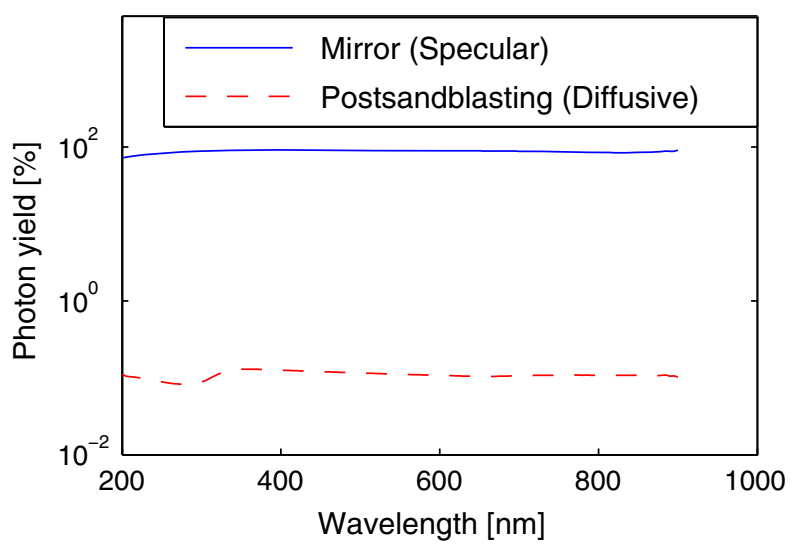

FIG. 10. Comparison of the relative photon yield from the polished and sandblasted regions of the target surface. 
chamber walls and beam pipe. This high field magnet has a bending radius of $34.82 \mathrm{~m}$, and its nearest end is $18.1 \mathrm{~m}$ from the target. A second set of vertical bumps was adjusted to move the angle of the emitted SR beam such that the light incident on the target was again minimized.

During this process, the SR intensity was monitored by imaging the target surface using the optical system. Generally, the signal-to-noise ratio was improved by minimizing the SR intensity incident on the target using the vertical orbit adjustments. Therefore, they were left in place for the entire experimental run.

\section{Target fabrication summary}

In Table IV, the measured parameters from each method of target fabrication are summarized. Through these investigations, it has been found that the optimum method of target fabrication, while ensuring a good coplanarity, flatness, and in-built SR suppression, is to sandblast the chemically etched silicon wafer such that there is a small specified reflective region of the target surface.

\section{EXPERIMENTAL RESULTS}

\section{A. Beam centering in the target aperture}

In order to maximize the lifetime during the beam size measurement, the electron beam must be centered within the target aperture. Direct imaging of the target surface was found to be the best method for this beam alignment.

Diffraction radiation is predominantly emitted from the edges of the target aperture. By comparing the intensity from each slit edge, the beam position relative to the center of the target aperture is obtained. An example of the peak asymmetry of the DR emitted by the target is shown in Fig. 11.

Using a closed vertical bump around the DR instrumentation in the storage ring, the beam position was adjusted such that the DR emission from the upper and lower target tines was equal, i.e. zero peak asymmetry. At this position, the electron beam is aligned to the center of the target aperture.

Close to the center of the target aperture, there is a linear relation between the beam position and peak asymmetry. In Fig. 12, the sensitivity in this central region is such that a micron change in the beam position corresponds to a $1 \%$ change in the peak asymmetry [27]. The error on the peak asymmetry is $\pm 2 \%$. Assuming the linear relation, this leads to a beam centering accuracy of $<5 \mu \mathrm{m}$.

This technique of beam centering has been applied for noninvasive optical beam positioning and was demonstrated at the KEK-ATF2 facility in Japan [27]. Using infrared wavelengths would allow a larger target aperture to be used. Increasing the slit size would further improve the beam lifetime, at the expense of vertical beam size sensitivity.

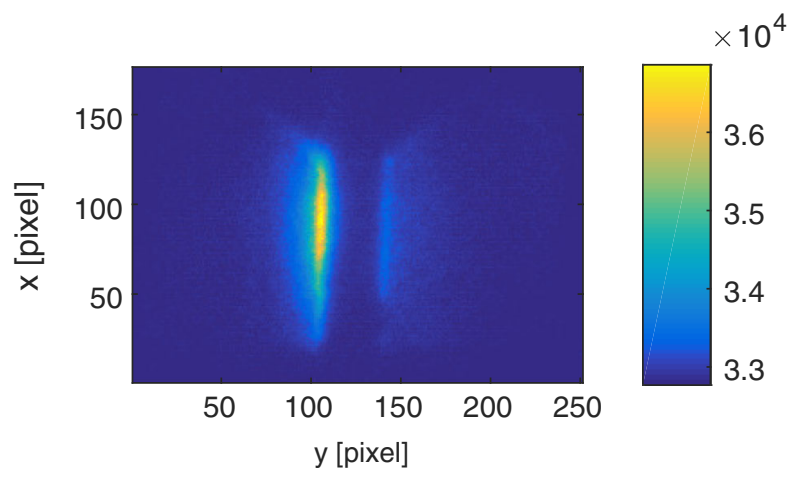

(a)

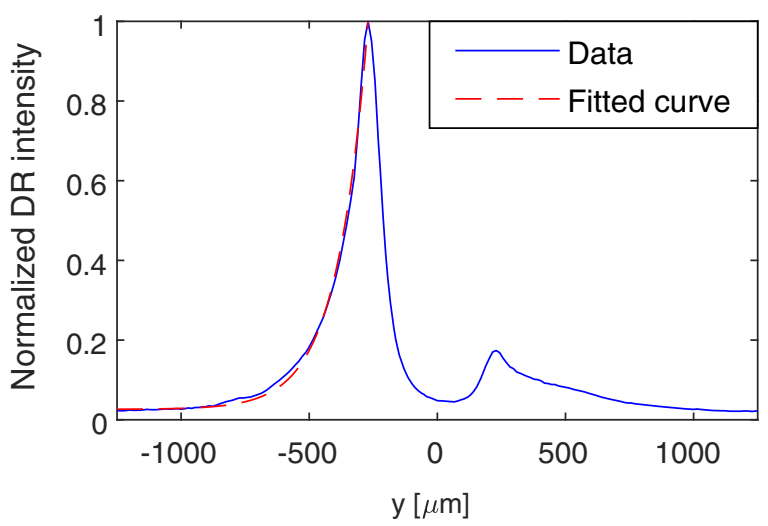

(b)

FIG. 11. Asymmetric light emission due to a $-52 \mu \mathrm{m}$ beam offset with respect to the center of the target aperture: (a) the intensity distribution across the target surface and (b) the corresponding line profile with a fitted exponential curve, characteristic of DR emission, of the form $f(y)=A \exp (B y)+C$, where $A=8.92, B=0.008$, and $C=0.026$. The target aperture is $500 \mu \mathrm{m}$.

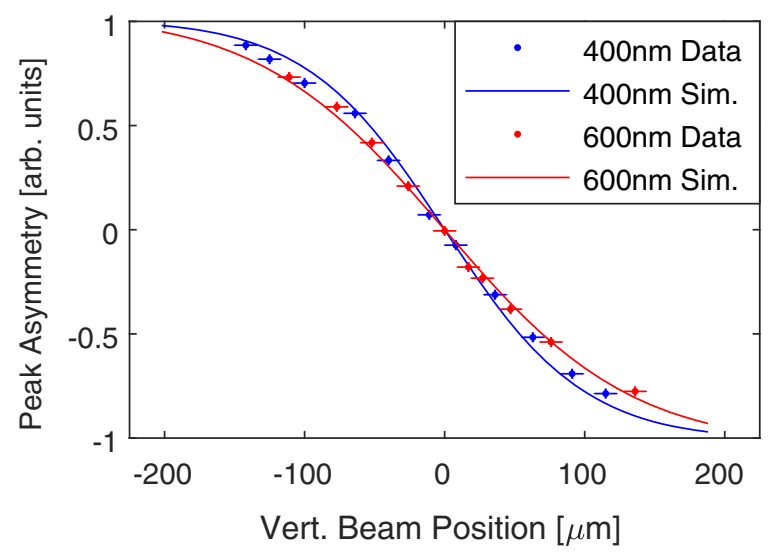

FIG. 12. Relation between the asymmetry of the DR intensity from the target tines and the beam position controlled by a vertical bump and measured by a nearby BPM. 


\section{B. Beam size measurement using the ODR model}

The angular distribution of DR for the noninterference setup using a $0.5 \mathrm{~mm}$ target and $2 \mathrm{~mm}$ mask is shown in Fig. 13. As expected, the central lobes have a significantly greater intensity compared to the side fringes, which are just visible.

The visibility $\left(I_{\min } / I_{\max }\right)$ of the DR angular distribution is sensitive to the beam size. The visibility can be measured from the central line profile or from the vertical projection of the angular distribution. The XBSM was used as the reference beam size monitor to which the DR measurements were compared. Beam sizes were measured using DR at 400 and $600 \mathrm{~nm}$ wavelengths. From the DR theory, as described in Sec. III, it is known that the sensitivity to the beam size scales inversely with the wavelength.

In Fig. 14, the beam size sensitivity is illustrated by the difference in intensity observed at $\theta_{y}=0 \mathrm{mrad}$ for different beam sizes. The visibility of the vertical projections at $46.2 \mu \mathrm{m}$ beam size are greater than that at $17.6 \mu \mathrm{m}$ as expected. The $46.2 \mu \mathrm{m}$ line profile with a known beam offset relative to the target center also has a visibility greater than that of the centered beam. Thus, in Fig. 14, the ambiguity and contribution due to the beam offset in the target aperture to the beam size measurement can be observed.

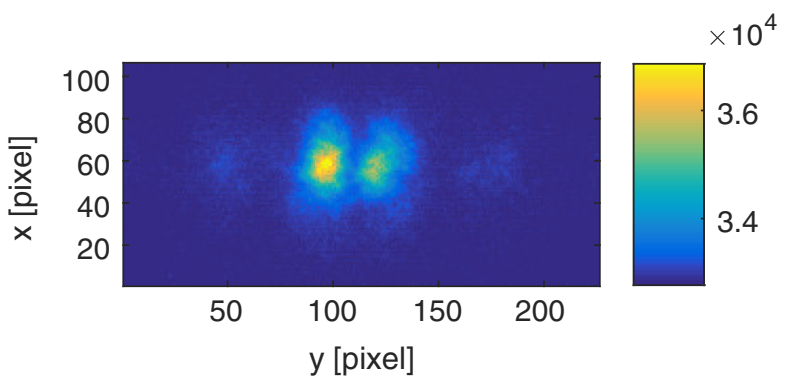

FIG. 13. An example of the DR angular distribution: $\lambda=600 \mathrm{~nm}, 0.5 \mathrm{~mm}$ target, and $2 \mathrm{~mm}$ mask.

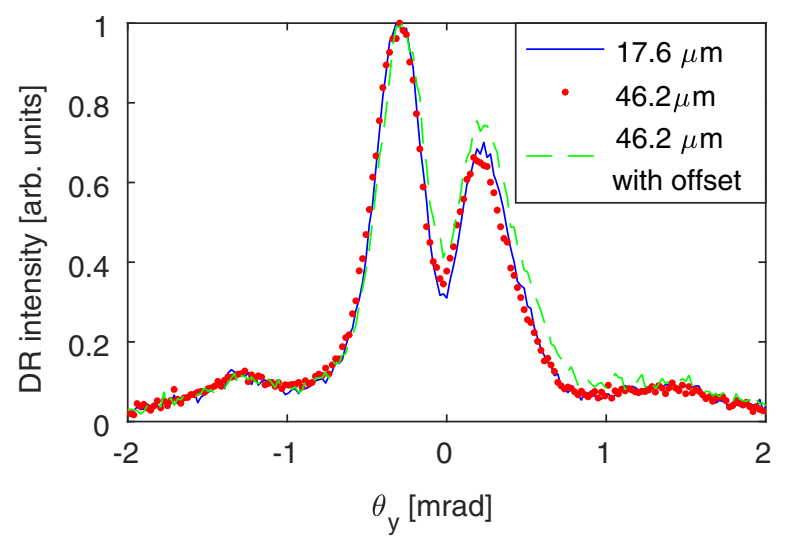

FIG. 14. A comparison of projected vertical polarization components for different beam sizes at $600 \mathrm{~nm}$.
The beam offset in the target aperture causes a change in the visibility only through the modification of $I_{\min }$ at the center of the angular distribution. It does not introduce any asymmetry of the lobes of the angular distribution. There are three potential causes of the lobe asymmetry in the acquired angular distribution: (i) The two tines of the target are not coplanar. As shown from the target metrology (see Table IV), the coplanarity of the tines of the target is less than $\lambda / 10$ and thus negligibly small [19]. (ii) There is an offset between the mask and target such that they are not collinear. If the destructive interference is significant when using a two-slit (mask and target) setup, the asymmetry should be constant and should not depend on the beam offset. In Sec. VIC, it is demonstrated that the angular distribution becomes more symmetric with a reduction of the mask aperture. Therefore, this indicates that the mask and target are sufficiently collinear. (iii) From the SR contribution, a residual part of the SR still propagates through the mask and is reflected by the target. This is likely to be the main source of the asymmetry in Fig. 14, since target imaging has shown that the SR is not uniformly distributed across the target surface (see Fig. 8).

Using the ODR model and PVPC technique, the expected visibility curve was simulated. Given a $600 \mathrm{~nm}$ wavelength and $0.5 \mathrm{~mm}$ target aperture size, for a range of beam sizes up to $50 \mu \mathrm{m}$, the angular distribution was simulated. From each angular distribution, the PVPC was obtained and the expected visibility for each beam size was recorded. The resulting visibility curve is shown in Fig. 15. The simulated visibility curve assumes a zero background contribution; thus, the curve passes through the origin.

The simulated visibility curve in Fig. 15 was fitted using the method of least squares with

$$
R=a_{0}+a_{1} \sigma_{y}+a_{2} \sigma_{y}^{2},
$$

where $R$ denotes the visibility, to obtain the coefficients $a_{0}$, $a_{1} \mathrm{~m}^{-1}$, and $a_{2} \mathrm{~m}^{-2}$. The coefficient $a_{0}$ defines the crossing point on the visibility axis. The fitted coefficients of the

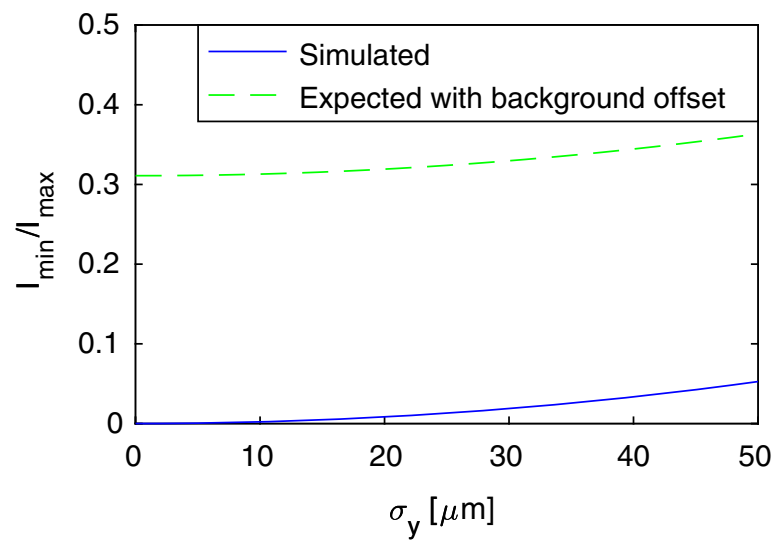

FIG. 15. Simulated (blue line) and expected (green dashed line) visibility curves for a $0.5 \mathrm{~mm}$ target and $600 \mathrm{~nm}$ wavelength. 
simulated visibility curve were $a_{0}=0.0, a_{1}=-15.49 \mathrm{~m}^{-1}$, and $a_{2}=2.13 \times 10^{7} \mathrm{~m}^{-2}$.

For real data measurements, there will be some background contribution to the DR distribution. Thus, $a_{0}$ will be greater than zero. Using the angular distribution from the real data at $17.6 \mu \mathrm{m}$ beam size, an estimation of the background offset was determined. From the theoretical curve in Fig. 15, it is seen that there is a very small difference in visibility between 0 and $17.6 \mu \mathrm{m}$ beam size. Thus, this estimation assumes that the background level is approximately the same as the measured visibility at $17.6 \mu \mathrm{m}$, which was 0.311 for the particular image that was analyzed. Therefore to obtain an estimation of the real visibility curve from the data, the 0.311 background was added to the theoretical curve obtained from the simulation, also shown in Fig. 15.

The difference in visibility between 17.6 and $46.2 \mu \mathrm{m}$ beam size using the ODR model simulation was 0.034 . The difference in visibility between 17.6 and $46.2 \mu \mathrm{m}$ beam size, using the simulated visibility curve including the background offset of 0.311, was 0.039 . Therefore, it was a reasonable assumption that only the background offset parameter $a_{0}$ in Eq. (8) needed to be adjusted to 0.311 for the estimated visibility curve of the real data.

To obtain a more accurate representation of the measured visibility curve at $600 \mathrm{~nm}$ wavelength, the average visibilities at beam sizes of 17.6 and $46.2 \mu \mathrm{m}$ were measured. To obtain the average visibility at each beam size, multiple images were analyzed using the ODR with PVPC technique. These average data points denoted by green crosses are shown in Fig. 16, where the error bars denote the standard error, which is 0.004 from $\leq 5$ images. Given this experimental error, it is recommended that the average visibility from multiple images should be used alongside the calibrated visibility curve to obtain a beam size measurement from the DR monitor.

The average visibility data points for 17.6 and $46.2 \mu \mathrm{m}$ were 0.2978 and 0.3380 , respectively. As aforementioned,

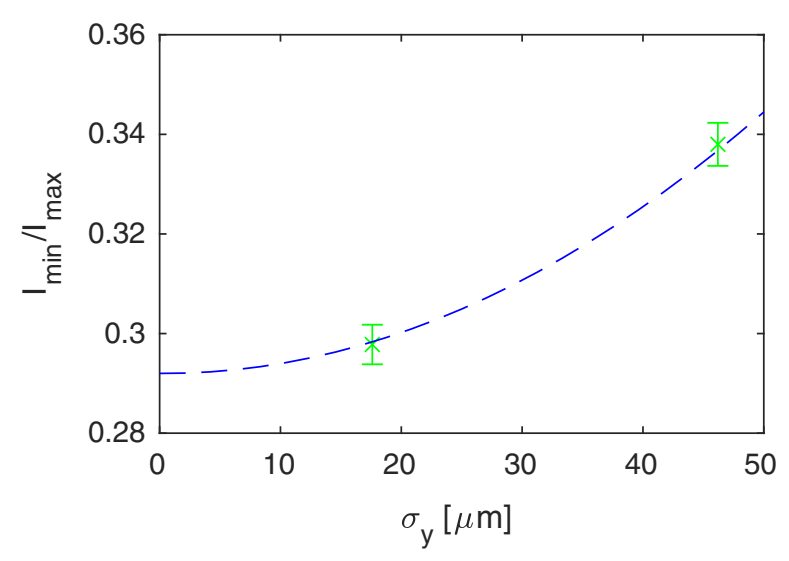

FIG. 16. Resultant visibility curve (dashed line) at $600 \mathrm{~nm}$ wavelength and $0.5 \mathrm{~mm}$ target aperture from a least squares fit of the average visibility at two beam sizes (green crosses). since the real data trend was in agreement with the quadratic behavior expected, as shown in Fig. 15, the coefficients $a_{1}$ and $a_{2}$ from the fit of the simulated curve with Eq. (8) were applied to the average visibility measurements. Therefore, given the average visibility data points and the previously obtained coefficients $a_{1}=-15.49 \mathrm{~m}^{-1}$ and $a_{2}=2.13 \times 10^{7} \mathrm{~m}^{-2}$ from the simulation, a least squares fit was performed with the two average data points to obtain an accurate value for the free parameter $a_{0}$.

In Fig. 16, the visibility curve from the least squares fit of the data is shown. Here the background offset for the real data was found to be $a_{0}=0.292$. This background offset is predominantly due to the SR still propagating through the mask aperture and reflected from the target.

Data were also acquired at $36.6 \mu \mathrm{m}$ beam size and $600 \mathrm{~nm}$ wavelength. At this beam size, the measured visibilities from two images were 0.346 and 0.341 . In comparison with the fitted visibility curve in Fig. 16, these values are extremely large compared to the expected value of approximately 0.32 . Unfortunately, from direct imaging of the target surface, it was found that the beam was not well centered in the target aperture. Because of this offset in the target aperture and the ambiguity between the beam size and beam offset, the measured beam size for this data set was increased and thus must be excluded from the fitted visibility curve.

From Fig. 16, it was found that the coefficient $a_{0}$ obtained from the least squares fit using the data was displaced from the expected value of zero predicted by the theory; i.e. the visibility should be zero for a vertical beam size of zero. The cause of the displacement of $a_{0}$ from zero is due to background SR which is captured with the DR angular distribution. At $600 \mathrm{~nm}$ wavelength, it was found that the SR contributed primarily as a background offset to the DR angular distribution and did not modify the DR interference fringes noticeably.

The vertical beam size sensitivity improves with a decreasing wavelength. However, in this experiment, the SR contribution increased with a decreasing wavelength such that at shorter wavelengths the signal-to-noise ratio is worsened. At $400 \mathrm{~nm}$, it was not possible to accurately measure the visibility due to the modification of the distribution due to SR.

In Fig. 17, the angular distribution of DR at $400 \mathrm{~nm}$ in the 0.5 and $2.0 \mathrm{~mm}$ target and mask apertures, respectively,

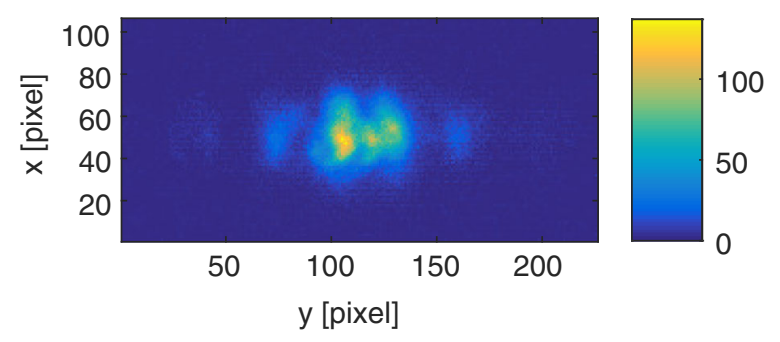

FIG. 17. DR angular distribution at $400 \mathrm{~nm}$ wavelength and $0.5 \mathrm{~mm}$ target aperture. 


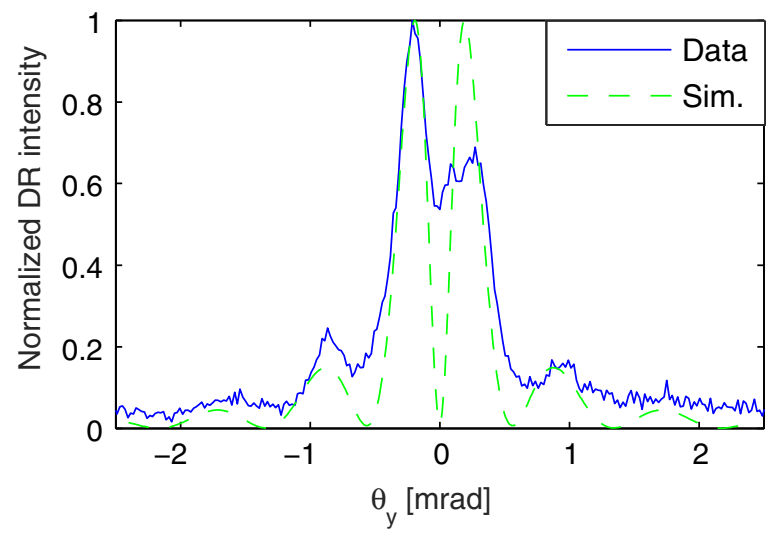

FIG. 18. Comparison of the $400 \mathrm{~nm}$ line profile from Fig. 17 and expected ODR distribution.

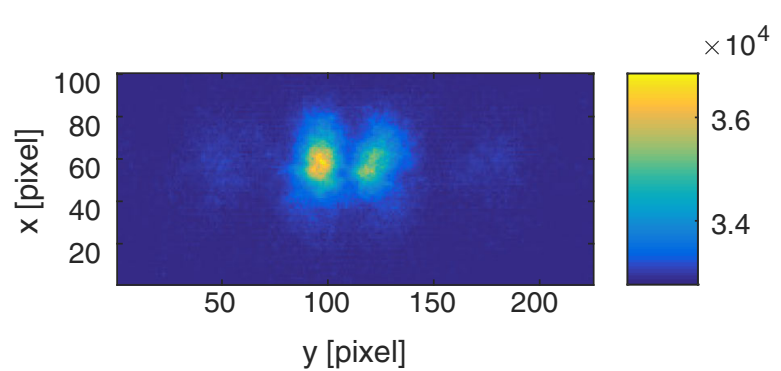

(a)

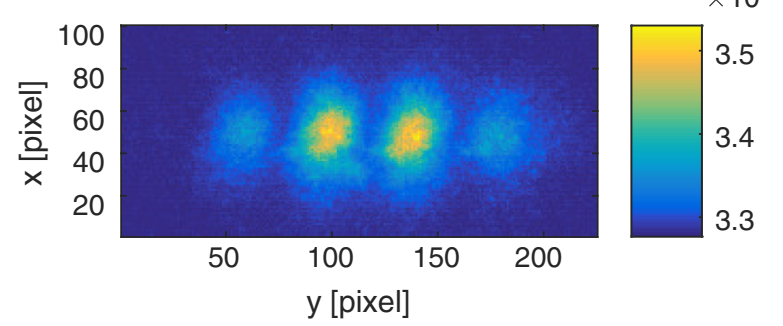

(b)

FIG. 19. Contour plots of the angular distributions at $600 \mathrm{~nm}$ for (a) ODR and (b) ODRI.

is shown. The central line profile from Fig. 17 is compared with the expected distribution from the ODR model in Fig. 18. In this case, the DR fringes have been distorted in amplitude and shape due to the interference with SR.

\section{Beam size measurement using the ODRI approach}

In Fig. 19, the ODR and ODRI angular distributions may be compared. Figure 19(a) shows the noninterference case
(ODR) using a $0.5 \mathrm{~mm}$ target and $2.0 \mathrm{~mm}$ mask. Figure 19(b) shows the interference case (ODRI) using a $1.0 \mathrm{~mm}$ mask. An enhancement of the side fringes is observed due to the interference between FDR and BDR from the mask and target, respectively.

To fit the beam size only, a reasonable estimate of the beam divergence was calculated as shown in Table VI. This was done by calculating the vertical beam emittance using the beam size measurement from the XBSM and the machine optics shown in Table V.

From the ODRI images, the central line profiles for different beam sizes were plotted against the expected distributions predicted by the ODRI model. In Figs. 20 and 21, the comparisons between the ODRI model and measured angular distributions for 17.6 and $36.6 \mu \mathrm{m}$ beam sizes are shown, respectively.

The asymmetry between the two central lobes in Fig. 20 can be fitted by adjusting the coplanarity offset parameter of the target tines in the ODRI model. The coplanarity offset required for this fit was $-40.0 \mathrm{~nm}$, which is comparable to the metrology measurements of the molecular adhesion targets reported in Table IV.

Given the beam size, divergence, and observation wavelength, the ODRI model predicted that the central minimum of the angular distributions should not be significantly displaced from zero. However, as seen in Figs. 20 and 21, the minima of the acquired line profiles are significantly greater than those expected from the theory. One possibility is that background SR was responsible for this difference between the theoretical prediction and acquired data.

A beam offset relative to the center of the target aperture may similarly distort the angular distribution. To quantify the beam position offset, the line profile in Fig. 20 was fitted using the method of least squares, with the beam position as the only fit variable; all other parameters were fixed according to the target metrology measurements, calculated beam divergence, and scaling of the beam size as reported by the XBSM. The result of the least squares fit is shown in Fig. 22. From this fit, the beam offset was found to be $120.0 \mu \mathrm{m}$.

Because of numerous degrees of freedom in the ODRI model, it was difficult to attribute differences observed in the angular distributions solely to a change in the vertical beam size. For example, with multiple fit parameters it is possible to converge on different solutions for the same angular distribution. Therefore, it is preferred to constrain or fix as many of the parameters as possible through

TABLE V. Twiss parameters used to scale the beam size from the XBSM to the ODR monitor in the storage ring.

\begin{tabular}{|c|c|c|c|c|c|c|c|c|c|}
\hline \multirow[b]{2}{*}{ Name } & \multirow[b]{2}{*}{$\mathrm{S}[\mathrm{m}]$} & \multicolumn{4}{|c|}{$X$} & \multicolumn{4}{|c|}{$Y$} \\
\hline & & Beta $[\mathrm{m}]$ & Phi [rad] & Eta $[\mathrm{m}]$ & Design orbit $[\mathrm{mm}]$ & Beta $[\mathrm{m}]$ & Phi [rad] & Eta $[\mathrm{m}]$ & Design orbit [mm] \\
\hline e- XBSM & 23.40 & 1.1 & 4.605 & 0.52 & 0 & 12.5 & 1.263 & 0.00 & 0 \\
\hline DET 48W & 379.40 & 41.7 & 44.412 & -0.13 & 0 & 7.86 & 28.729 & 0.00 & 0 \\
\hline
\end{tabular}


TABLE VI. Table of the expected beam size and divergence at the ODR monitor as from the XBSM reference.

\begin{tabular}{lccc}
\hline \hline$\sigma_{y}(\mathrm{XBSM})[\mu \mathrm{m}]$ & $\varepsilon_{y}[\mathrm{~m}]$ & $\sigma_{y}(\mathrm{ODR})[\mu \mathrm{m}]$ & $\sigma_{y}^{\prime}(\mathrm{ODR})[\mu \mathrm{rad}]$ \\
\hline 22.2 & $3.06 \mathrm{E}-11$ & 17.6 & 4.08 \\
46.1 & $1.70 \mathrm{E}-10$ & 36.6 & 8.46 \\
58.2 & $2.71 \mathrm{E}-10$ & 46.2 & 10.7 \\
\hline \hline
\end{tabular}

optimization of the design and metrology, as well as simultaneous measurement of the beam position and beam size.

\section{Beam lifetime}

With the target retracted, the beam lifetime is approximately $20 \mathrm{~min}$, dominated by the Touschek effect. The beam lifetime in the storage ring with the target inserted reduced to 2-3 min using target apertures of 0.5 and $1.0 \mathrm{~mm}$.

The beam lifetime was stable at 2-3 min with the target inserted until the vertical beam size was increased to

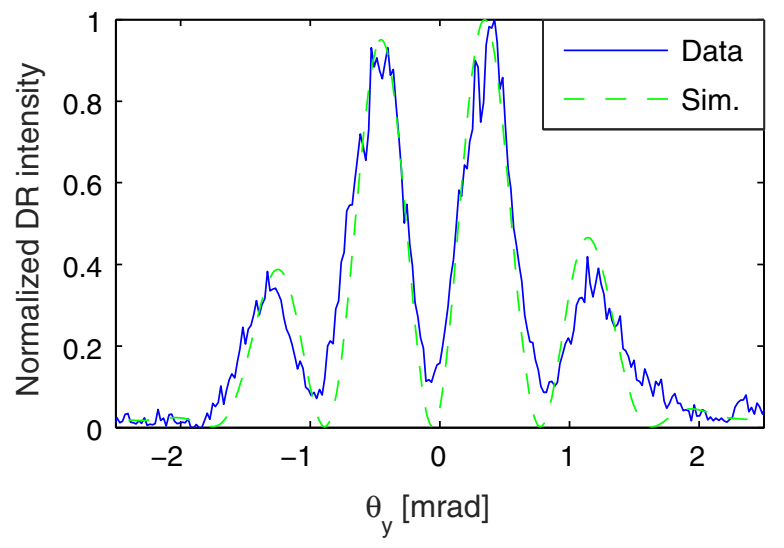

FIG. 20. ODRI line profile at $600 \mathrm{~nm}$ wavelength for $\sigma_{y}=17.6 \mu \mathrm{m}, \sigma_{y}^{\prime}=4.08 \mu \mathrm{rad}$.

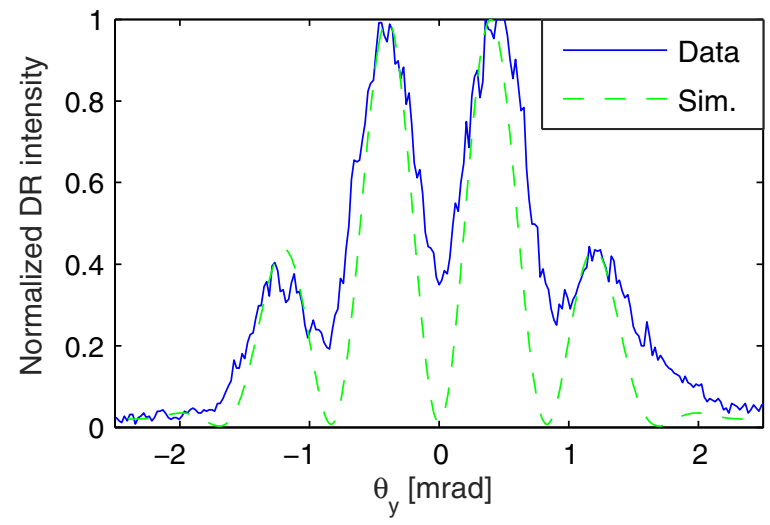

FIG. 21. ODRI line profile at $600 \mathrm{~nm}$ wavelength for $\sigma_{y}=36.6 \mu \mathrm{m}, \sigma_{y}^{\prime}=8.46 \mu \mathrm{rad}$.

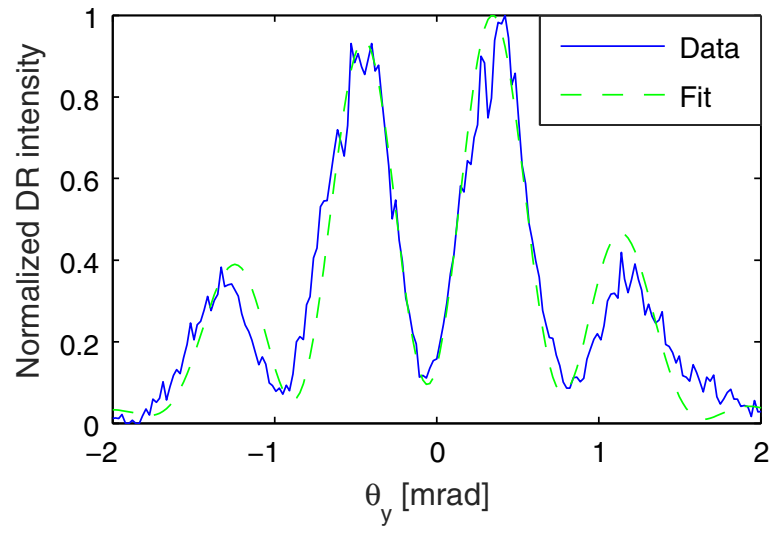

FIG. 22. A beam offset of $120 \mu \mathrm{m}$ obtained using a least squares fit for ODRI data with the beam offset in the target as the only fit variable.

$50 \mu \mathrm{m}$. At this size, the beam lifetime could be regained by manually adjusting the vertical beam position in the slit using target imaging, i.e. ensuring the beam is well centered in the target.

The impact of the target on the beam lifetime was unknown before this investigation. From scraper experiments, the vertical beam profile was measured to consist of a Gaussian core with exponential tails. These tails are enhanced by the insertion of the target. The fundamental cause of this tail growth is unknown.

It was also verified that scraping of the horizontal tails which extend much further than the vertical tails did not impact the beam lifetime. This was done by adjusting the skew (or tilt) of the beam passing through the target.

Furthermore, it was observed that the beam lifetime is not dependent on the charge per bunch. The beam lifetime observed for $1 \mathrm{~mA}$ single bunch is the same as for a $1 \mathrm{~mA}$ 10 bunch train. Thus, the reduction in lifetime is unlikely to be a wakefield effect.

From these observations, it is postulated that the tail growth is generated by beam gas scattering. This process is not dependent on the beam current and would exhibit the reduction in the beam lifetime that is observed.

As aforementioned, to improve the sensitivity of the DR monitor to the vertical beam size, the size of the target aperture could be decreased. However, from these investigations it has been found that the beam lifetime would be significantly affected through any reduction of the slit size.

\section{CONCLUSIONS}

In summary, the feasibility of DR for noninvasive, micron-scale, transverse beam size measurement in circular machines has been investigated. A DR monitor was designed, installed, and commissioned at CesrTA with a specific emphasis on the beam conditions and requirements for operation on the electron storage ring.

Significant developments in the target design and fabrication have been made. This includes a comparison of the 
well-established process of chemical etching used in physics applications with the relatively unknown molecular adhesion technique. Furthermore, investigations have shown the benefit of restricting the reflective area and sandblasting the target such that the two-slit (mask and target) setup can be reduced to a single-slit target with inbuilt masking of SR. From these results, the optimal target would be fabricated using molecular adhesion to best match the specified aperture size and coplanarity requirements, with the reflective areas restricted and sandblasting of the remaining polished surface. However, due to the fragility concerns of these targets and the cost, recent developments using the chemical etching procedure provide a more robust option. From this work, the fabrication technology developed in this experiment has already been applied to the DR instrumentation under test at the KEKATF2 facility in Japan [27].

Using a $0.5 \mathrm{~mm}$ target aperture, the sensitivity to a beam size variation between 18 and $46 \mu \mathrm{m}$ has been demonstrated at a $600 \mathrm{~nm}$ wavelength. For these measurements, a SR monitor (XBSM) was used as the reference. Beam size measurements were also performed at $400 \mathrm{~nm}$; however, it was found that the angular distribution was modified by the increased SR background.

The angular distributions were compared to two analytical models: the ODR model, which excludes the interference effect between the mask and target, and the ODRI model, where this interference is accounted for. Results have shown a good agreement between the data with those expected from the theory, especially once the SR background is accounted for. Once the visibility curve is calibrated with a reference monitor, the beam size measurement is very straightforward. Given the developments in target technology and simultaneous measurement of the beam position inside the slit, the authors are confident that the ODR model is applicable in a wide range of situations, providing reliable and unambiguous measurements of the beam size.

Direct imaging of DR on the target surface has demonstrated the application of beam position monitoring using DR. The beam centering accuracy in the target was measured as $<5 \mu \mathrm{m}$. Because of the ambiguity between the beam size and offset in the target in the angular distribution, it is recommended that a beam splitter is incorporated in the optical system such that the beam position and beam size are measured simultaneously. From the simulation of the visibility curves, for a $5 \mu \mathrm{m}$ beam offset in the slit aperture, the maximum error on the beam size measurement is $5 \mu \mathrm{m}$ at a $600 \mathrm{~nm}$ wavelength and $0.5 \mathrm{~mm}$ target aperture.

A significant reduction of the beam lifetime has been observed with the target inserted. The cause of this reduction is insensitive to the bunch current. It is suspected that growth of the vertical tails occurs via beam-gas scattering.

From these results, the use of DR monitors on circular electron machines is restricted. In this case, SR monitors are more suitable, provided source points are available. However, DR monitors may be useful on the transfer lines in the injector chain. Furthermore, the use of several DR monitors on large-scale linear accelerators and extraction lines should be further investigated.

The use of DR monitors may also be considered on high energy hadron machines which have demonstrated beam lifetimes in excess of $10 \mathrm{~h}$ while using tight collimator apertures of $6 \sigma_{x, y}$ to $9 \sigma_{x, y}$ [32]. On these machines, the beam sizes are considerably larger than those of electron beams, such that the target aperture can be increased and the required beam size sensitivity can be achieved using infrared wavelengths. Alternatively, the target aperture may be increased in future electron accelerators where the beam energy is significantly increased.

\section{ACKNOWLEDGMENTS}

The authors thank the technical departments of the Cornell Laboratory of Accelerator Sciences and Education (CLASSE), Cornell University, USA, and CERN, Switzerland, for their assistance in the assembly and installation of the instrument. Thanks must be given to N. Chritin for his contribution in the design stages and the provision of the technical drawings to the project. The authors also thank H. Schmickler, M. Palmer, and D. Rubin for the organization of the collaboration, as well as R. Jones for his continued support.

[1] M. L. Ter-Mikaelian, High-Energy Electromagnetic Processes in Condensed Media (Wiley-Interscience, New York, 1972).

[2] A. P. Potylitsyn, M. I. Ryazanov, M. N. Strikhanov, and A. A. Tishchenko, Diffraction Radiation from Relativistic Particles (Springer, Berlin, 2010).

[3] M. Castellano, A new non-intercepting beam size diagnostics using diffraction radiation from a slit, Nucl. Instrum. Methods Phys. Res., Sect. A 394, 275 (1997).

[4] P. Karataev, S. Araki, R. Hamatsu, H. Hayano, T. Muto, G. Naumenko, A. Potylitsyn, N. Terunuma, and J. Urakawa, Diffraction radiation from a charged particle moving through a rectangular hole in a rectangular screen, Nucl. Instrum. Methods Phys. Res., Sect. B 227, 198 (2005).

[5] R. B. Fiorito and D. W. Rule, Diffraction radiation diagnostics for moderate to high energy charged particle beams, Nucl. Instrum. Methods Phys. Res., Sect. B 173, 67 (2001).

[6] A. Potylitsyn et al., Coherent radiation recoil effect for the optical diffraction radiation beam size monitor at SLAC FFTB, Nucl. Instrum. Methods Phys. Res., Sect. B 227, 170 (2005).

[7] I. E. Vnukov, B. N. Kalinin, G. A. Naumenko, D. V. Padalko, A. P. Potylitsyn, and O. V. Chefonov, Experimental observation of optical diffraction radiation, J. Exp. Theor. Phys. 67, 802 (1998).

[8] T. Muto, S. Araki, R. Hamatsu, H. Hayano, T. Hirose, P. Karataev, G. Naumenko, A. Potylitsyn, and J. Urakawa, 
Observation of Incoherent Diffraction Radiation from a Single-Edge Target in the Visible-Light Region, Phys. Rev. Lett. 90, 104801 (2003).

[9] P. Karataev, S. Araki, R. Hamatsu, H. Hayano, T. Muto, G. Naumenko, A. Potylitsyn, N. Terunuma, and J. Urakawa, Observation of optical diffraction radiation from a slit target at KEK accelerator test facility, Nucl. Instrum. Methods Phys. Res., Sect. B 227, 158 (2005).

[10] P. Karataev, S. Araki, A. Aryshev, G. Naumenko, A. Potylitsyn, N. Terunuma, and J. Urakawa, Experimental observation and investigation of the prewave zone effect in optical diffraction radiation, Phys. Rev. ST Accel. Beams 11, 032804 (2008).

[11] P. Karataev, S. Araki, R. Hamatsu, H. Hayano, T. Muto, G. Naumenko, A. Potylitsyn, N. Terunuma, and J. Urakawa, Beam-Size Measurement with Optical Diffraction Radiation at KEK Accelerator Test Facility, Phys. Rev. Lett. 93, 244802 (2004).

[12] E. Chiadroni, M. Castellano, A. Cianchi, K. Honkavaara, G. Kube, V. Merlo, and F. Stella, in Proceedings of the 22nd Particle Accelerator Conference, PAC-2007, Albuquerque, NM (IEEE, New York, 2007), p. 3982.

[13] A. H. Lumpkin, J. W. Berg, N. S. Sereno, D. W. Rule, and C. Y. Yao, Near-field imaging of optical diffraction radiation generated by a 7-GeV electron beam, Phys. Rev. ST Accel. Beams 10, 022802 (2007).

[14] M. Aicheler, P. Burrows, T. Draper, T. Garvey, P. Lebrun, K. Peach, N. Phinney, H. Schmickler, D. Schulte and N. Toge, CERN Technical Report No. CERN-2012-007, 2012.

[15] A. Hansson, E. Walln, and X. Andersson, Transverse electron beam imaging system using visible synchrotron radiation at MAX III, Nucl. Instrum. Methods Phys. Res., Sect. A 671, 94 (2012).

[16] L. J. Nevay, R. Walczak, and L. Corner, High power fiber laser system for a high repetition rate laserwire, Phys. Rev. ST Accel. Beams 17, 072801 (2014).

[17] P. V. Karataev, Pre-wave zone effect in transition and diffraction radiation: Problems and solutions, Phys. Lett. A 345, 428 (2005).

[18] V. A. Verzilov, Transition radiation in the pre-wave zone, Phys. Lett. A 273, 135 (2000).

[19] P. Karataev, Ph.D. thesis, Tokyo Metropolitan University, 2004.

[20] P. Karataev, R. Hamatsu, T. Muto, S. Araki, H. Hayano, J. Urakawa, G. Naumenko, A. Potylitsyn, and T. Hirose, in
Proceedings of the Joint 28th Advanced Beam Dynamics and Advanced and Novel Accelerator Workshop, QABP'03 (2004), p. 111, http://inspirehep.net/record/638527?ln=en.

[21] A. Cianchi, M. Castellano, L. Catani, E. Chiadroni, K. Honkavaara, and G. Kube, Nonintercepting electron beam size monitor using optical diffraction radiation interference, Phys. Rev. ST Accel. Beams 14, 102803 (2011).

[22] K. Lekomtsev, G. A. Blair, G. Boorman, R. Corsini, P. Karataev, T. Lefevre, and M. Micheler, Coherent diffraction radiation experiment at CTF3-Simulation studies, Il Nuovo Cimento C 34, 261 (2011).

[23] M. Billing, The conversion of CESR to operate as the test accelerator, CesrTA. Part 1: overview, J. Instrum. 10, P07012 (2015).

[24] J. P. Alexander et al., Vertical beam size measurement in the CESR-TA $e^{+} e^{-}$storage ring using $\mathrm{x}$-rays from synchrotron radiation, Nucl. Instrum. Methods Phys. Res., Sect. A 748, 96 (2014).

[25] S. T. Wang, D. L. Rubin, J. Conway, M. Palmer, D. Hartill, R. Campbell, and R. Holtzapple, Visible-light beam size monitors using synchrotron radiation at CESR, Nucl. Instrum. Methods Phys. Res., Sect. A 703, 80 (2013).

[26] M. Billing et al., Beam position monitoring system at CESR, J. Instrum. 12, T09005 (2017).

[27] R. Kieffer et al., Optical diffraction radiation for position monitoring of charged particle beams, Nucl. Instrum. Methods Phys. Res., Sect. B 402, 88 (2017).

[28] M. S. Kulkarni and H. F. Erk, Acid-based etching of silicon wafers: Mass-transfer and kinetic effects, J. Electrochem. Soc. 147, 176 (2000).

[29] S. Franssila, Anisotropic Wet Etching, Introduction to Microfabrication (Wiley, New York, 2010), pp. 237-254, ISBN 9781119990413.

[30] S. Kerdiles, C. Duret, A. Vaufredaz, and F. Metral, Process for Bonding by Molecular Adhesion (2012), http://www .google.com/patents/US8158013.

[31] P. Karataev, H. Hayano, T. Muto, N. Terunuma, J. Urakawa, R. Hamatsu, G. Naumenko, and A. Potylitsyn, in Proceedings of the 9th European Particle Accelerator Conference, Lucerne, 2004 (EPS-AG, Lucerne, 2004).

[32] S. Redaelli, R. Assmann, and G. Robert-Demolaize, LHC aperture and commissioning of the collimation system, in Proceedings of Chamonix 2005 LHC Project Workshop (2005), p. 268. 